\title{
Second and third harmonic measurements at the linac coherent light source
}

\author{
D. Ratner, ${ }^{1}$ A. Brachmann, ${ }^{2}$ F. J. Decker, ${ }^{2}$ Y. Ding, ${ }^{2}$ D. Dowell, ${ }^{2}$ P. Emma, ${ }^{2}$ A. Fisher, ${ }^{2}$ J. Frisch, ${ }^{2}$ S. Gilevich, ${ }^{2}$ \\ Z. Huang, ${ }^{2}$ P. Hering, ${ }^{2}$ R. Iverson, ${ }^{2}$ J. Krzywinski, ${ }^{2}$ H. Loos, ${ }^{2}$ M. Messerschmidt, ${ }^{2}$ H. D. Nuhn, ${ }^{2}$ \\ T. Smith, ${ }^{2}$ J. Turner, ${ }^{2}$ J. Welch, ${ }^{2}$ W. White, ${ }^{2}$ and J. $\mathrm{Wu}^{2}$ \\ ${ }^{1}$ Department of Applied Physics, Stanford University, 348 Via Pueblo Mall, Stanford, California 94305, USA \\ ${ }^{2}$ SLAC National Accelerator Laboratory, 2575 Sand Hill Road, Menlo Park, California 94025, USA
}

(Received 9 May 2011; published 2 June 2011)

\begin{abstract}
The linac coherent light source (LCLS) is a self-amplified spontaneous emission (SASE) free-electron laser (FEL) operating at fundamental photon energies from 0.5 to $10 \mathrm{keV}$. Characterization of the higher harmonics present in the FEL beam is important to users, for whom harder $\mathrm{x}$ rays can either extend the useful operating wavelength range or increase experimental backgrounds. We present measurements of the power in both the second and third harmonics, and compare the results to expectations from simulations. We also present studies of the transport of harmonics to the users, and the harmonic power as a function of electron beam quality.
\end{abstract}

DOI: 10.1103/PhysRevSTAB.14.060701

PACS numbers: 41.60.Cr, 42.55.Vc, 41.50.+h

\section{INTRODUCTION}

The linac coherent light source (LCLS) started user commissioning in October of 2009, producing fundamental free-electron laser (FEL) radiation with photon energies ranging from 550 to $10 \mathrm{keV}$ [1]. Radiation at the fundamental wavelength of the FEL dominates in the experimental beam lines, but non-negligible levels of radiation at higher harmonics are also present. These harmonics may be desirable as a source of harder $\mathrm{x}$ rays, but may also contribute backgrounds to user experiments at the fundamental wavelength.

The self-amplified spontaneous emission (SASE) FEL interaction introduces both energy and density modulations of the electron beam at the undulator's fundamental wavelength. Close to saturation, strong bunching at this wavelength produces rich harmonic bunching as well [2]. Three-dimensional simulations [3] and analytical results [4] show that significant odd harmonic power can exist for a planar undulator at the FEL saturation. Previous shortwavelength SASE FEL experiments, such as those at VISA and FLASH, have observed third harmonic emission as high as $1 \%$ of the fundamental power $[5,6]$.

While the harmonic bunching factor is largest at the second harmonic, the symmetry of planar undulators prohibits on-axis radiation at even harmonics, so we expect the third harmonic to dominate the harmonic emission. However, due to finite electron beam size, betatron motion, and radiation angle, second harmonic radiation may exist at non-negligible levels $[3,7,8]$, and has also been observed experimentally at LEUTL, VISA, and FLASH $[5,6,9]$.

Published by the American Physical Society under the terms of the Creative Commons Attribution 3.0 License. Further distribution of this work must maintain attribution to the author(s) and the published article's title, journal citation, and DOI.
In this paper we present measurements of the secondand third harmonic content in the FEL at a range of photon energies. To determine the extent to which higher harmonics reach the experimental stations, we measure the photon energy cutoff of the $\mathrm{x}$-ray mirrors. We also compare the results to simulations and study the effect of electron beam quality on the harmonic power.

\section{DATA COLLECTION METHODS}

The primary goal of this paper is to determine the relative pulse energy levels of the harmonics in the FEL beam at LCLS. To measure the harmonic content, we separate the FEL components by photon energy. In standard operation, the sum of all higher harmonics represents at most a few percent of the FEL beam, so we take the total intensity measurements as an approximate measure of the fundamental pulse energy. We then use mirrors and attenuators to separate out the second and third harmonics, which we measure by inserting YAG screens into the $\mathrm{x}$-ray beam. We measure the third harmonic at both soft and hard $\mathrm{x}$ rays, and the second harmonic at soft $\mathrm{x}$ rays only.

\section{A. Third harmonic method, soft and hard $x$ rays}

To measure the third harmonic, we attenuate the x-ray beam with either $\mathrm{N}_{2}$ gas or solid sheets of beryllium or zirconium (Fig. 1). The attenuation decreases at shorter wavelengths, allowing harmonic radiation to pass while blocking the fundamental. As an example, with the fundamental set to $8 \mathrm{keV}$ photons, the zirconium filter ( $1 \mathrm{~mm}$ of silicon with $100 \mu \mathrm{m}$ of zirconium) cuts the fundamental x-ray intensity by 10 orders of magnitude, but cuts the third harmonic by less than 2 orders of magnitude [10]. The attenuator does not suppress higher harmonics, but these are emitted at much lower levels in 


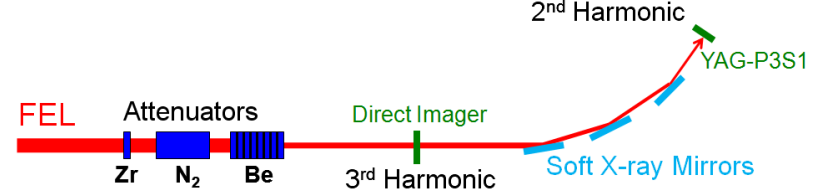

FIG. 1. Schematic of the harmonic measurements. Attenuators block the fundamental and second harmonic, allowing measurement of the third harmonic on the direct imager YAG screen. Alternatively, we can isolate the second harmonic by measuring the intensity at the P3S1 YAG screen; the soft x-ray mirrors, upstream of P3S1, absorb the third harmonic. We again rely on the attenuators to block the fundamental.

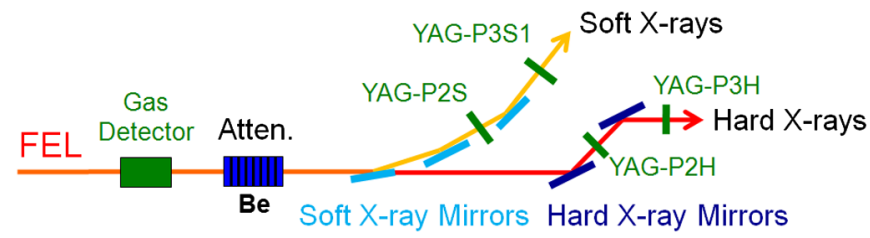

FIG. 2. Schematic of the mirror cutoff measurement. For the soft $x$-ray line, the ratio of intensities measured on YAG screens $\mathrm{P} 3 \mathrm{~S} 1$ and P2S gives the transmission for a single mirror, and the total transmission is assumed to be the cube of this ratio. Alternatively, we can compare the intensity on P3S1 (downstream of all mirrors) directly against the intensity at the gas detector (upstream of all mirrors). For the hard x-ray line, attenuators block the fundamental, and we calculate the square of the ratio of third harmonic measured before $(\mathrm{P} 3 \mathrm{H})$ and after $(\mathrm{P} 2 \mathrm{H})$ the second mirror.

the FEL process, so we assume the radiation remaining past the attenuator is primarily third harmonic. We can also independently confirm the presence of the third harmonic by tuning the FEL to the zirconium $\mathrm{K}$ edge (Sec. III B).

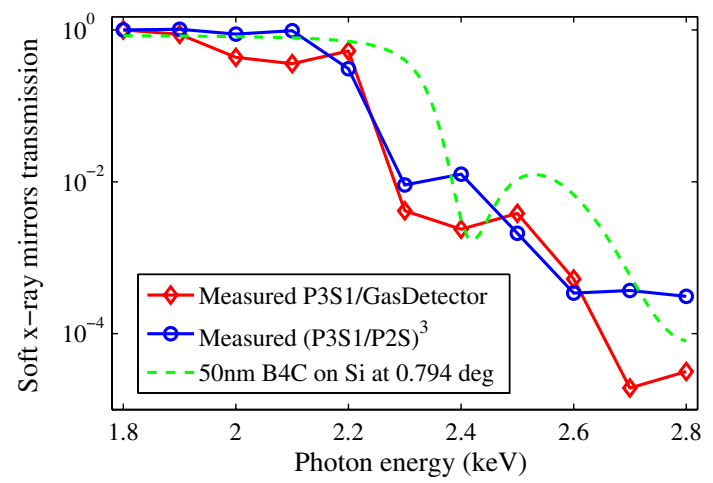

\section{B. Second harmonic method, soft $x$ rays}

We measure the second harmonic, sandwiched between the stronger first and third harmonics, in the experimental beam lines. Solid or gas attenuators again block the fundamental, letting only higher harmonics pass. However, the mirrors that direct the radiation to the experimental beam lines absorb most radiation above a cutoff photon energy. By setting the FEL fundamental photon energy to between $1 / 3$ and $1 / 2$ of the cutoff, the mirrors will pass the second harmonic while absorbing the third harmonic (Fig. 1). With the low energy photons absorbed in the attenuators, and the high energy photons absorbed in the mirrors, only the second harmonic reaches the experimental beam lines. Because of the gradual energy cutoff for the hard x-ray mirrors (Fig. 3), the second harmonic measurements work only for soft $\mathrm{x}$ rays.

\section{Intensity measurement methods}

We obtain x-ray intensities from either a gas detector or a YAG screen [11]. For each YAG image, we average up to 100 pulses from the FEL. Though we subtract a dark (no electron beam) background, spontaneous radiation is present in all images. To determine the FEL pulse intensity, we fit a 2D Gaussian profile to the YAG image and calculate the volume under the FEL peak. Because the spontaneous radiation emits at a much wider angle than the FEL, we assume the spontaneous components appear as a pedestal underneath the FEL peak. We then expect the volume under the curve to represent the FEL pulse energy only.

\section{Transport mirror cutoff}

To determine the level of second harmonic reaching the experimental stations, we measure the photon cutoff energy of the beam line mirrors. A series of three glancing

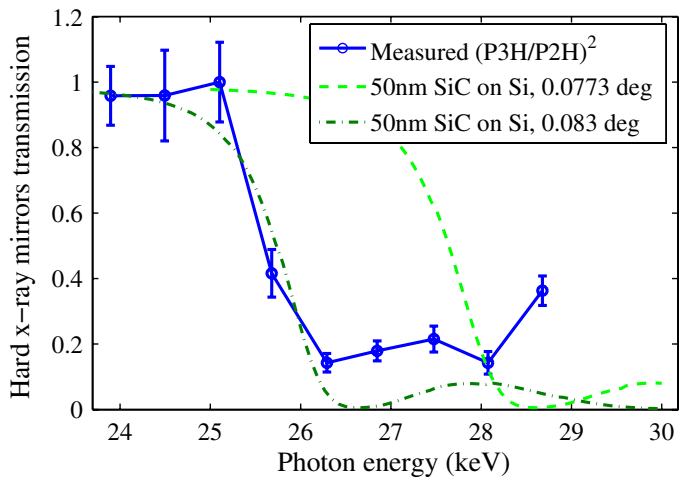

FIG. 3. Transmission plots show the photon energy cutoffs of the soft x-ray (left plot) and hard x-ray (right plot) experimental beam lines. On the left, blue circles show the cube of the ratio of intensities on P3S1 and P2S, while the red diamonds give the ratio from P3S1 to the full pulse energy. Both results match the expected transmission (dashed green curve) [10,12]. On the right, blue circles give the square of the ratio of intensities measured on $\mathrm{P} 3 \mathrm{H}$ and $\mathrm{P} 2 \mathrm{H}$. The dashed curve gives the expected transmission at the nominal $0.0773^{\circ}$ angle; however, we find a better match for $0.083^{\circ}$ angle (dot-dashed curve). Hard x-ray measurements rely on the third harmonic, resulting in lower resolution than for the soft x-ray measurements. In both cases, the experimental curves are normalized to one at the maximal value. 
incidence mirrors diverts the x-ray beam to the soft x-ray experimental halls. The mirrors absorb hard $\mathrm{x}$-ray radiation, which cannot reach the soft x-ray experimental stations. YAG screens before and after the third mirror (P2S and P3S1, respectively) measure the transmitted $\mathrm{x}$-ray pulse energy. We determine the mirror cutoff energy by two methods. First, we measure the ratio of intensities on P3S1 and P2S as a function of photon energy. Assuming all three mirrors are identical, we plot the cube of this ratio as the total transmission of the mirrors. However, the transmission of each stage may differ, for example if the mirror aperture cuts a portion of the FEL beam. As a semiindependent method, we also compare the signal on P3S1 to the total incoming power measured in the gas detectors, located upstream of the mirrors. Figure 2 diagrams both methods, and we plot the results in Fig. 3, showing a cutoff energy of approximately $2.3 \mathrm{keV}$ as expected [12].

A set of two mirrors directs high energy radiation to the hard x-ray hutches. The photon energy cutoff of the hard $\mathrm{x}$-ray line is far beyond the limit of the fundamental FEL, so we insert an attenuator that allows only third harmonic radiation to pass. We again vary the photon energy while comparing the third harmonic power seen before and after the final mirror (using screens $\mathrm{P} 2 \mathrm{H}$ and $\mathrm{P} 3 \mathrm{H}$, respectively). We compare the measured and expected values in Fig. 3 [13]. The discrepancy between the curves is likely the result of a change in the angle of the mirrors. With a cutoff approximately a factor of 3 above the maximum energy of the fundamental, we expect all of the second harmonic to reach the hard x-ray experimental stations. Third harmonic radiation up to $25 \mathrm{keV}$ will also pass through to the hard $\mathrm{x}$-ray lines.

\section{THIRD HARMONIC MEASUREMENTS, SOFT AND HARD X RAYS}

\section{A. Attenuator scan}

To measure the third harmonic, we block the fundamental with either beryllium or zirconium attenuators. (The attenuation also blocks the weaker second harmonic.) To find the relative power of the third harmonic, we can simply take the ratio of intensities on the YAG screen with a sufficiently thick attenuator inserted (primarily third harmonic) and removed (primarily fundamental). For 0.9 and $1.7 \mathrm{keV}$ fundamental photons, we find approximately $2 \%$ and $3 \%$ harmonic content, respectively.

We can also vary the level of attenuation, and fit the harmonic content to the resulting curve. By measuring the transmission values for the filters, we simultaneously confirm the validity of the harmonic measurement. The total intensity is proportional to

$$
\begin{aligned}
I(d) \propto & T_{1}(d) P_{1}+T_{2}(d) P_{2}+T_{3}(d) P_{3} \\
& + \text { higher harmonics, }
\end{aligned}
$$

where, for harmonic $h$, we have attenuator transmission $T_{h}(d)$ and FEL power $P_{h}$. We assume that the transmission can be described by a simple exponential,

$$
T_{h}(d)=e^{-d / \Lambda_{h}}
$$

with the attenuation length $\Lambda_{h}$ determined by the attenuator composition, the fundamental photon energy, and the harmonic of interest. The attenuator parameter $d$ can be either the thickness of a solid attenuator (units of $\mathrm{mm}$ ) or the pressure in a gas attenuator (units of torr). The attenuation length $\Lambda_{h}$ then has units of either mm for the solid attenuator or torr for the gas attenuator. Neglecting the weaker second and higher harmonics, we expect

$$
I(d) \propto e^{-d / \Lambda_{1}}+e^{-d / \Lambda_{3}} \frac{P_{3}}{P_{1}}
$$

Measuring the intensity $I(d)$ for a range of $d$, we can then find the ratio of the harmonics $P_{3} / P_{1}$ from a one-parameter, linear fit.

The beryllium filter transmission is difficult to estimate from the composition of the filters; even low levels of impurities of heavy elements can have a large impact on the absorption of hard $\mathrm{x}$ rays. To confirm the transmission values, we repeat the analysis with a three-parameter nonlinear fit of both attenuation lengths $\Lambda_{1}, \Lambda_{3}$ as well as the harmonic ratio $P_{3} / P_{1}$. For data sets without multiple measurements per attenuator setting, we assume a measurement error of $5 \%$ for each data point. To estimate error bars for the fit, we perform a Monte Carlo simulation, randomizing each data point according to its error and repeating the fit. We find the attenuation lengths at the fundamental $\Lambda_{1}$ match the expected values reasonably well (Table I). Because of the long attenuation lengths for the third harmonic at high photon energies, the errors on $\Lambda_{3}$ are large. (However, we note that the harmonic ratio $P_{3} / P_{1}$ is not sensitive to $\Lambda_{3}$.)

At lower photon energies, we assume the YAG response to the fundamental and third harmonic is equivalent.

TABLE I. Nominal (middle column) and measured (right column) attenuation lengths for the fundamental and third harmonic at three different photon energies. The nominal and fitted attenuation lengths match well for the fundamental, but in general the fitted third harmonic values are lower than expected. Fitting for the third harmonic attenuation length at hard $\mathrm{x}$ rays is not effective due to the low absorption of the third harmonic.

\begin{tabular}{lcc}
\hline \hline Photon energy & Nominal $\Lambda_{1}, \Lambda_{3}$ & Nonlinear fit $\Lambda_{1}, \Lambda_{3}$ \\
\hline $0.9 \mathrm{keV}$ & $0.33,7.42 \mathrm{torr}$ & $0.38 \pm 0.02,9.4 \pm 1.8 \mathrm{torr}$ \\
$6 \mathrm{keV}$ & $2.0,21 \mathrm{~mm}$ & $1.9 \pm 0.1,12.5 \pm 3.3 \mathrm{~mm}$ \\
$8 \mathrm{keV}$ & $3.8,28 \mathrm{~mm}$ & $3.33 \pm 0.30, \ldots \mathrm{mm}$ \\
$9 \mathrm{keV}$ & $5.1,30 \mathrm{~mm}$ & $5.2 \pm 0.1, \ldots \mathrm{mm}$ \\
\hline \hline
\end{tabular}



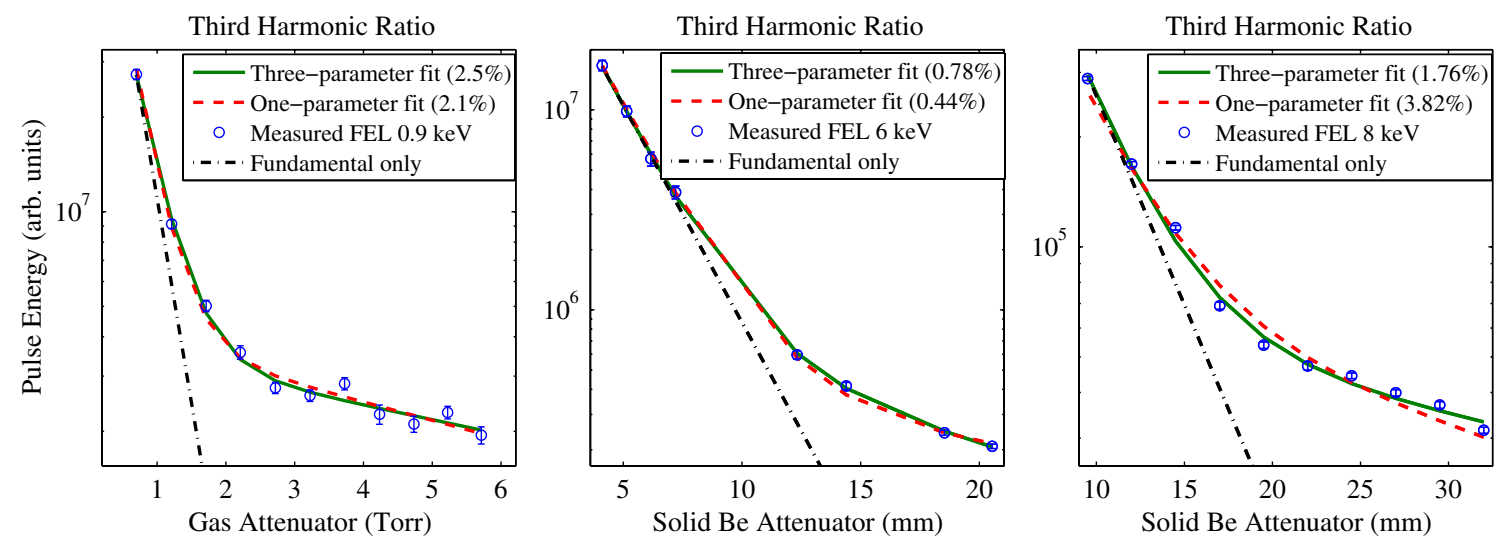

FIG. 4. Examples of third harmonic measurements for fundamental FEL photon energies of $0.9 \mathrm{keV}$ (left), $6 \mathrm{keV}$ (center), and $8 \mathrm{keV}$ (right). Blue circles show the experimental data, red dotted lines show linear one-parameter fits using the nominal attenuation lengths, while solid green lines show three-parameter fits to both the harmonic content and attenuation lengths. The black dot-dashed line shows the expected drop in intensity for the fundamental only. We typically find around $2 \%$ third harmonic for soft $\mathrm{x}$ rays, with between $0.2 \%-2 \%$ third harmonic at hard x rays. The $4 \%$ level found from the one-parameter fit at $8 \mathrm{keV}$ (right plot) is higher than expected, and may be artificially high due to a lack of measurement points dominated by the fundamental.

However, as the photon energy increases, the YAG screen may not fully absorb the third harmonic. For the $6 \mathrm{keV}$ fundamental measurement, a $100 \mu \mathrm{m}$ YAG largely absorbs the third harmonic due to the yttrium $\mathrm{K}$ edge at $17 \mathrm{keV}$. At $8 \mathrm{keV}$ fundamental, approximately $40 \%$ of the third harmonic passes through the $100 \mu \mathrm{m}$ YAG, so we use a $1 \mathrm{~mm}$ YAG for this measurement.

Figure 4 shows several sample measurements, with $0.8 \%-2 \%$ third harmonic content at a range of photon energies. In general, we find slightly higher harmonic content with the fundamental set to longer wavelengths. However, the harmonic content at any particular wavelength may vary widely depending on the electron beam

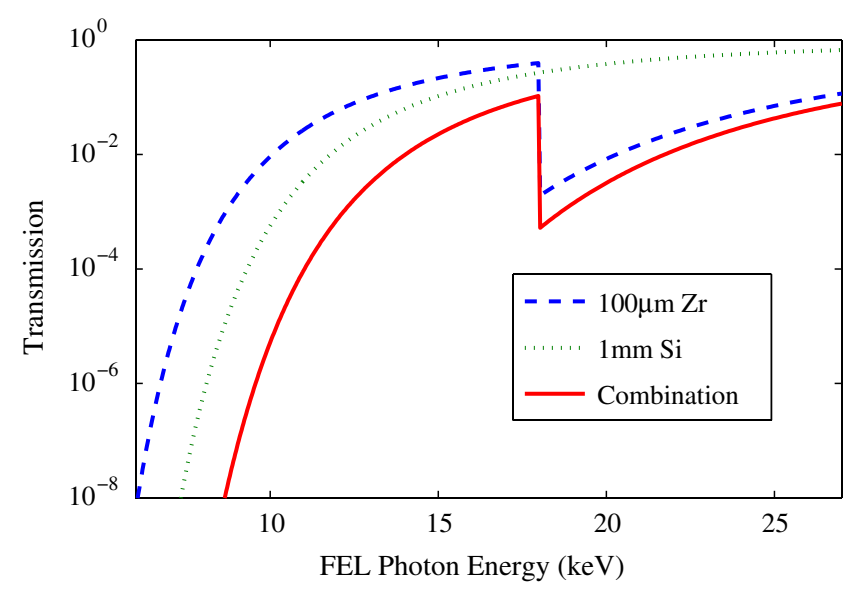

FIG. 5. Transmission for the zirconium filter as a function of photon energy. The filter is made of $100 \mu \mathrm{m}$ of zirconium (dashed blue) and $1 \mathrm{~mm}$ of silicon (dotted green), with the combined transmission given in solid red. The zirconium $\mathrm{K}$ edge manifests as a sharp drop in transmission at $18 \mathrm{keV}$. parameters (e.g., slice emittance, alignment in the undulator line) at the time of each measurement.

\section{B. K-edge scan}

With the FEL fundamental photon energy tuned to $6 \mathrm{keV}$, we can confirm the third harmonic measurement by inserting a zirconium filter and scanning the FEL photon energy around the $\mathrm{K}$ edge (Fig. 5). A small shift in energy of a few percent has little effect on the FEL performance, but changes the zirconium transmission at $18 \mathrm{keV}$ by more than 2 orders of magnitude; we conclude that any drop in measured intensity must come from

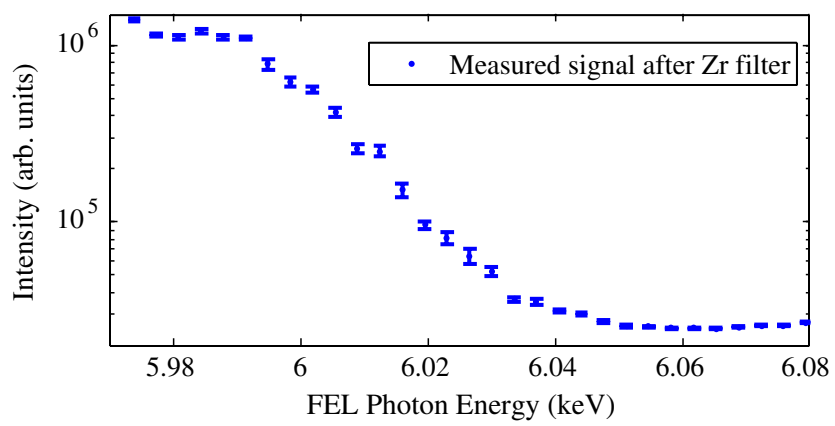

FIG. 6. Plot of measured intensity on the direct imager YAG screen ( $y$ axis) vs photon energy ( $x$ axis), with the FEL tuned to near $6 \mathrm{keV}$ fundamental and the zirconium attenuator inserted. The dropoff in intensity as the fundamental photon energy crosses $6 \mathrm{keV}$ corresponds to the zirconium $\mathrm{K}$ edge at $18 \mathrm{keV}$; we conclude that the drop in intensity is due to third harmonic content. With the zirconium filter blocking the fundamental, we find that the remaining radiation is almost entirely third harmonic. Electron energy jitter and FEL bandwidth broaden the otherwise sharp $\mathrm{K}$ edge seen in Fig. 5. 
radiation at the $18 \mathrm{keV} \mathrm{K}$ edge. With the zirconium filter inserted, the intensity drops by more than a factor of 40 across the K edge (Fig. 6), so we conclude that below the K edge the measured signal consists almost entirely of third harmonic.

To determine the third harmonic content, we set the third harmonic photon energy $300 \mathrm{eV}$ below the $\mathrm{K}$ edge, and compare the signal with the zirconium filter inserted (primarily third harmonic) and removed (combination of third harmonic and fundamental). To prevent YAG and camera saturation during the fundamental measurement, we insert a $4 \mathrm{~mm}$ Be filter. We can then find the harmonic content from the ratio of the beryllium $\left(I^{(\mathrm{Be})}\right)$ and zirconium $\left(I^{(\mathrm{Zr})}\right)$ signals:

$$
\frac{I^{(\mathrm{Be})}}{I^{(\mathrm{Zr})}}=\frac{T_{1}^{(\mathrm{Be})} P_{1}+T_{3}^{(\mathrm{Be})} P_{3}}{T_{1}^{(\mathrm{Zr})} P_{1}+T_{3}^{(\mathrm{Zr})} P_{3}} \approx \frac{T_{1}^{(\mathrm{Be})}}{T_{3}^{(\mathrm{Zr})}} \frac{P_{1}}{P_{3}}+\frac{T_{3}^{(\mathrm{Be})}}{T_{3}^{(\mathrm{Zr})}},
$$

with power $P_{h}$ and transmission factors $T_{h}$ for both the zirconium and beryllium attenuators at harmonic $h$. Plugging in the known transmission values (the beryllium filter transmits $8 \%$ fundamental and nearly $100 \%$ third harmonic), we find approximately $2 \%$ third harmonic content, confirming the results of Fig. 4.

\section{SECOND HARMONIC MEASUREMENTS, SOFT X RAYS}

We expect weaker second harmonic than third harmonic due to the symmetry of the planar LCLS undulators.

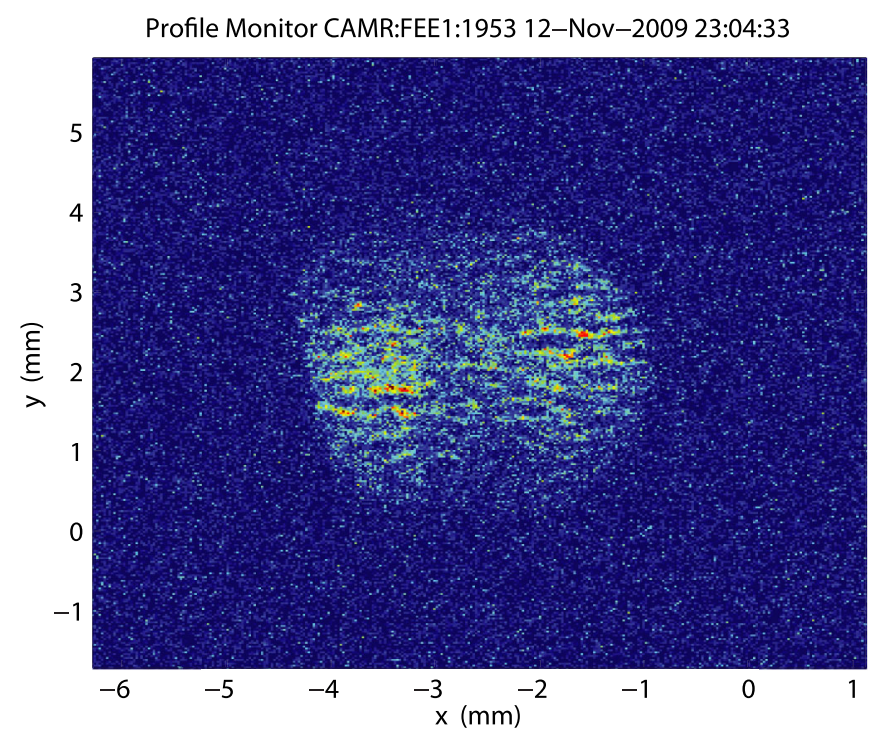

FIG. 7. An example image in the soft x-ray beam line (P3S1) shows the characteristic double lobe structure of the second harmonic. Though gas and solid (beryllium) attenuators strongly suppress the fundamental in this image, a small amount of fundamental radiation remains (Gaussian mode background). Diffraction from the beryllium attenuators produces the uneven speckle pattern.
Though on-axis radiation is suppressed at even harmonics, the finite electron beam size and betatron oscillations lead to non-negligible second harmonic content $[3,7,8]$. (We note that the bunching factor, a measure of the longitudinal correlation of particles, is stronger for the second harmonic. Tuning a group of "afterburner" undulators to double the resonant frequency can exploit this bunching to produce even more power at the second harmonic than would otherwise exist at the third harmonic [14]. In this paper we assume the undulators are tuned to the fundamental.) By measuring the FEL beam intensity following the soft x-ray mirrors, we are able to isolate the second harmonic. An example image (Fig. 7) shows the characteristic double lobe structure expected for the second harmonic (e.g., [8]).

To measure the second harmonic component, we again vary the attenuation and fit the ratio $P_{2} / P_{1}$ from the intensity,

$$
\begin{aligned}
I & \propto e^{-d / \Lambda_{1}} M_{1}^{3}+e^{-d / \Lambda_{2}} M_{2}^{3} \frac{P_{2}}{P_{1}}+e^{-d / \Lambda_{3}} M_{3}^{3} \frac{P_{3}}{P_{1}} \\
& \approx e^{-d / \Lambda_{1}}+e^{-d / \Lambda_{2}} \frac{P_{2}}{P_{1}},
\end{aligned}
$$

where we have assumed the mirror transmission $M_{h}$ is perfect for the fundamental and second harmonic, and zero for the third harmonic (Fig. 3).

The second harmonic measurements are especially sensitive to the $\mathrm{N}_{2}$ transmission value at the fundamental. When the attenuation is strongest, the harmonic dominates and the attenuation length at the fundamental has little effect. However, at the lowest attenuation levels when the fundamental dominates, the $\mathrm{N}_{2}$ still provides as many as six attenuation lengths at the fundamental. As a result, even small errors in the attenuation length lead to large errors in the measured power level. (The need for heavy attenuation is due to the small dynamic range of the P3S1 camera, which was not intended for this use.)

To confirm the attenuation lengths, we again fit the measured intensities to two generic exponentials. The measured and nominal attenuation lengths match reasonably well at the fundamental, but differ substantially at the second harmonic (Table II). Figure 8 gives sample results for the second harmonic content.

TABLE II. Nominal (middle column) and measured (right column) second harmonic attenuation lengths for the gas detector. The nominal and fitted attenuation lengths match well for the fundamental, but in general the fitted second harmonic values are lower than expected.

\begin{tabular}{lcc}
\hline \hline Photon energy & Nominal $\Lambda_{1}, \Lambda_{2}$ & Nonlinear fit $\Lambda_{1}, \Lambda_{2}$ \\
\hline $0.9 \mathrm{keV}$ & $0.31,2.15$ torr & $0.28 \pm 0.02,1.54 \pm 0.08$ torr \\
$1 \mathrm{keV}$ & $0.42,2.95$ torr & $0.44 \pm .07,2.27 \pm .47$ torr \\
\hline \hline
\end{tabular}



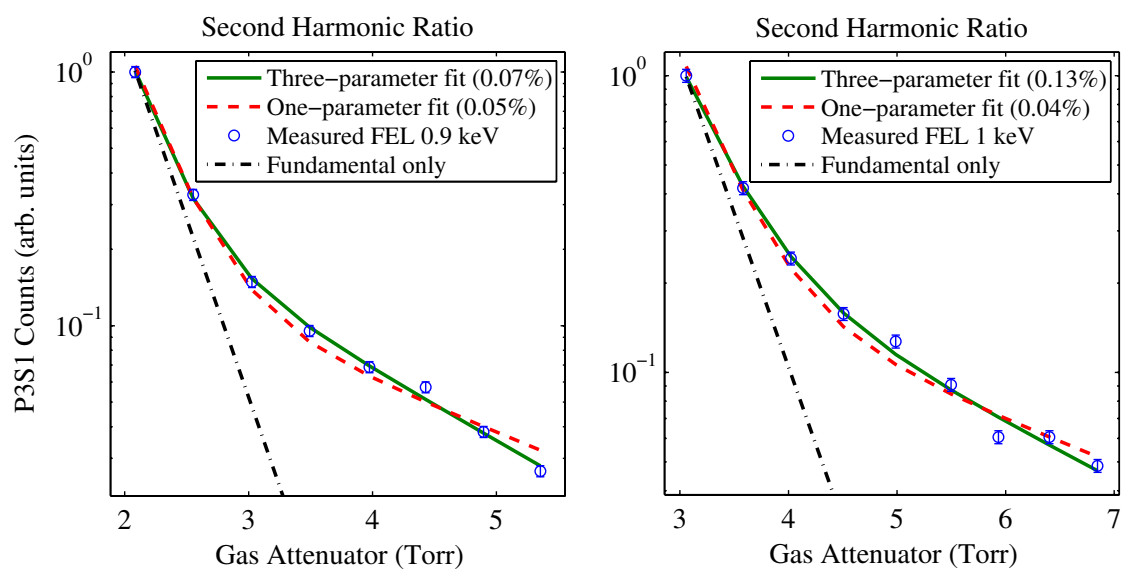

FIG. 8. We measure the harmonic component by varying the gas attenuator strength for $0.9 \mathrm{keV}$ (left) and $1 \mathrm{keV}$ (right) fundamental photon energies. Blue circles show experimental data, red dotted lines show one-parameter fits using the nominal attenuation lengths, and solid green lines show three-parameter fits to both the harmonic content and attenuation lengths. The black dot-dashed line shows the expected attenuation of the fundamental only. We find approximately $0.05 \%-0.1 \%$ second harmonic content.

\section{GENESIS SIMULATIONS}

We compare our results to simulations from the 3D wiggler-averaged code GENESIS [15], using the parameters of Table III. Simulations at FEL saturation predict approximately $3 \%$ third harmonic at soft $\mathrm{x}$ rays $(830 \mathrm{eV}$ in simulation), and approximately $2 \%$ third harmonic at hard $\mathrm{x}$ rays $(8.3 \mathrm{keV}$ in simulation). For both energies, the fifth harmonic is approximately an order of magnitude weaker in simulations, and thus could contribute slightly to the third harmonic measurements. (We have not tried to separate the third harmonic from the higher harmonic contributions.) We conclude that the measured third harmonic content is approximately consistent with the level expected from theory and simulations, albeit with weaker harmonics than expected at high energies $[4,16]$. Second harmonic levels from GENESIS are also in approximate agreement with results. However, GENESIS is a wiggler-averaged code (the beam centroid does not move off-axis), so comparisons with second harmonic simulations require further study.

\section{SENSITIVITY TO BEAM QUALITY}

We expect the proportion of harmonics present to vary depending on the performance of the fundamental. The

TABLE III. GENESIS simulation parameters given for soft and hard x-ray simulations. The simulations are consistent with measurements for both soft and hard $\mathrm{x}$ rays.

\begin{tabular}{lcc}
\hline \hline & Soft x rays & Hard x rays \\
\hline Photon energy & $0.83 \mathrm{keV}$ & $8.3 \mathrm{keV}$ \\
Electron energy & $4.3 \mathrm{GeV}$ & $13.6 \mathrm{GeV}$ \\
Emittance $(x, y$, normalized) & $0.4 \mu \mathrm{m}$ & $0.4 \mu \mathrm{m}$ \\
Peak current & $1 \mathrm{kA}$ & $1 \mathrm{kA}$ \\
Electron beam energy spread & $3 \times 10^{-4}$ & $1 \times 10^{-4}$ \\
Third harmonic content & $3 \%$ & $2 \%$ \\
\hline \hline
\end{tabular}

lowest and highest harmonic contents in Fig. 4 were measured with 0.6 and $1.5 \mathrm{~mJ}$ fundamental pulse energy, respectively. Because of the increased sensitivity to electron bunch quality at shorter wavelengths, the correlation between low fundamental pulse energy and low harmonic content is not surprising. (Low fundamental energy may indicate decreased bunching factor at the fundamental, which would have a larger effect on the harmonics.)

To test the sensitivity of harmonics to electron beam quality, we repeat the third harmonic measurements while intentionally degrading the electron beam. A convenient method for manipulating the electron beam is the laser heater, which can increase the electron energy spread in a simple and measurable fashion (Fig. 9) [17]. We

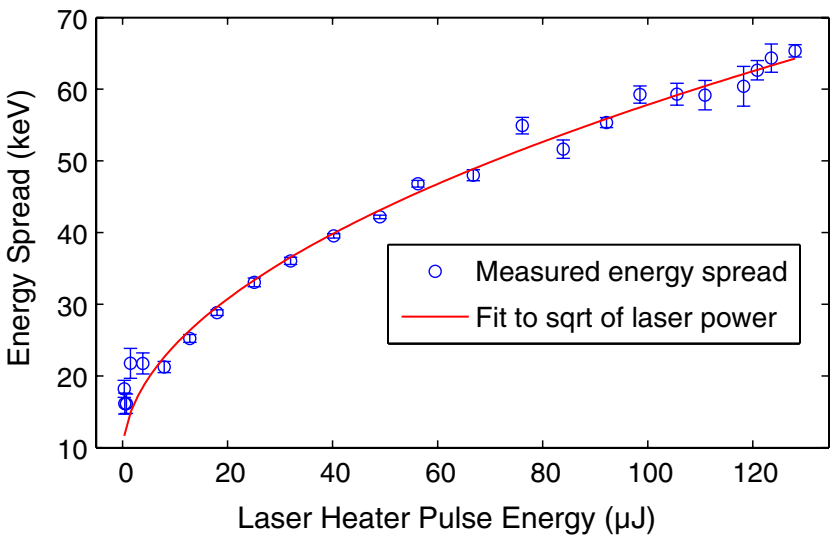

FIG. 9. Energy spread measured as a function of laser heater power. To determine energy spread, we measure the electron beam width in the dispersive region a few meters downstream from the laser heater. By increasing the energy of the laser heater, we are able to triple the energy spread of the electron beam. The energy spread increases as the square root of the laser heater power, except at low laser power where trickle heating causes a noticeable increase in the energy spread [17]. 


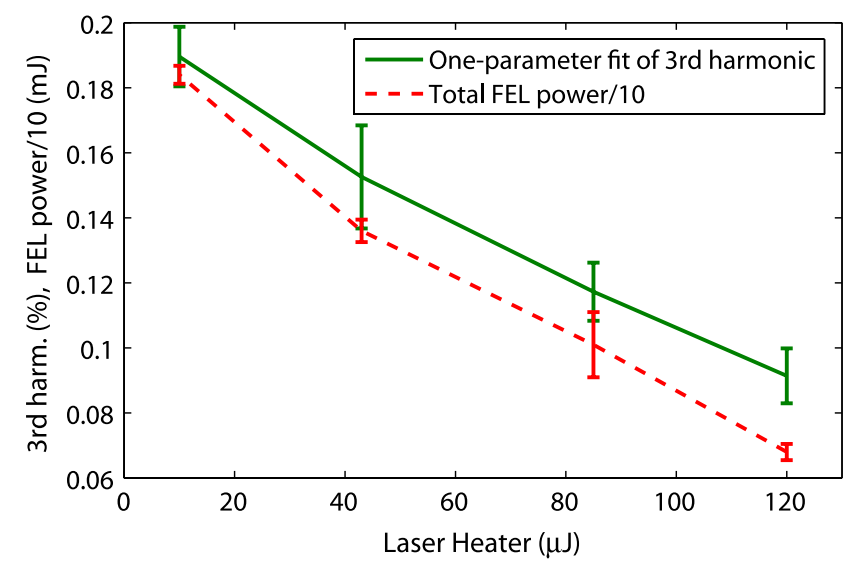

FIG. 10. Percentage of harmonic power vs laser heater setting. As the FEL performance drops (dotted red line), the percentage of third harmonic also drops (solid green), indicating enhanced sensitivity to electron beam quality at higher harmonics. The FEL performance was determined by energy loss scans, which measure the energy loss of the electrons due to the FEL process [1].

then measure the $8 \mathrm{keV}$ third harmonic content at each of the four laser heater strengths. To ensure that we operate in the saturation region, we insert all 28 available undulators for each measurement and confirm that we reach saturation [18].

We find that as the FEL performance drops (determined by the total power in the FEL beam), the proportion of power due to the third harmonic also drops (Fig. 10). By increasing the laser heater above the nominal setting, users may be able to suppress unwanted harmonics.

We note that even at the nominal heating level, the harmonic content only reached $0.2 \%$. The low harmonic level may be related to the poor FEL performance on the day of the measurement; though the fundamental $\mathrm{x}$-ray pulse energy was a respectable $2 \mathrm{~mJ}$, the FEL spot was highly non-Gaussian, suggesting misalignment of the electron beam in the undulator line. The higher sensitivity to electron beam quality of the third harmonic may explain the low harmonic percentage.

\section{CONCLUSION}

We present second and third harmonic measurements for LCLS (Table IV). At low energies (below $1 \mathrm{keV}$ fundamental) we measure less than $0.1 \%$ second harmonic content. The second harmonic reaches the soft x-ray beam line for fundamental photon energies below approximately $1.15 \mathrm{keV}$ (cutoff around $2.3 \mathrm{keV}$ ). At low and high energies, we measure third harmonic content ranging from $0.2 \%$ to $2.5 \%$, which is consistent with expectations. We confirm that the proportion of third harmonic falls as the FEL performance degrades.
TABLE IV. Summary of harmonic results. The second harmonic content in normal operation at soft $\mathrm{X}$-ray wavelengths is below $0.1 \%$. The third harmonic content is as high as about $2 \%$ at normal operation, but can drop by an order of magnitude or more due to poor electron beam quality.

\begin{tabular}{lcc}
\hline \hline Photon energy & 2nd harmonic & 3rd harmonic \\
\hline Soft x rays (near 1 keV) & $0.04 \%-0.1 \%$ & $2.0 \%-2.5 \%$ \\
Hard x rays $(6-8 \mathrm{keV})$ & & $0.2 \%-2 \%$ \\
\hline \hline
\end{tabular}

\section{ACKNOWLEDGMENTS}

We would like to thank S. Biedron and D. Zhu for helpful comments and all members of the LCLS commissioning team for making this work possible. This work is supported by Department of Energy Contract No. DEAC02-76SF00515.

[1] P. Emma et al., Nat. Photon. 4, 641 (2010).

[2] R. Bonifacio, L. D. Salvo, and P. Pierini, Nucl. Instrum. Methods Phys. Res., Sect. A 293, 627 (1990).

[3] H. Freund, S. Biedron, and S. Milton, IEEE J. Quantum Electron. 36, 275 (2000).

[4] Z. Huang and K.-J. Kim, Phys. Rev. E 62, 7295 (2000).

[5] A. Tremaine et al., Phys. Rev. Lett. 88, 204801 (2002).

[6] W. Ackermann et al., Nat. Photon. 1, 336 (2007).

[7] G. Geloni, E. Saldin, E. Schneidmiller, and M. Yurkov, DESY Report No. 05-137, DESY, 2005.

[8] Z. Huang and K. J. Kim, Nucl. Instrum. Methods Phys. Res., Sect. A 475, 112 (2001).

[9] S. Biedron et al., Nucl. Instrum. Methods Phys. Res., Sect. A 483, 94 (2002).

[10] http://henke.lbl.gov/optical_constants/.

[11] J. Welch et al., in Proceedings of the 32nd International Free Electron Laser Conference (FEL2010), Malmo, Sweden (2010).

[12] R. Soufli et al., Proc. SPIE Int. Soc. Opt. Eng. 7077, 707716 (2008).

[13] A. Barty, R. Soufli, T. McCarville, S. Baker, M. Pivovaroff, P. Stefan, and R. Bionta, Opt. Express 17, 15508 (2009).

[14] H. D. Nuhn et al., in Proceedings of the 32nd International Free Electron Laser Conference (FEL2010), Malmo, Sweden (2010).

[15] S. Reiche, Nucl. Instrum. Methods Phys. Res., Sect. A 429, 243 (1999).

[16] E. L. Saldin, E. A. Schneidmiller, and M. V. Yurkov, Phys. Rev. ST Accel. Beams 9, 030702 (2006).

[17] Z. Huang et al., Phys. Rev. ST Accel. Beams 13, 020703 (2010).

[18] D. Ratner et al., in Proceedings of the 31st International Free Electron Laser Conference (FEL 09), Liverpool, UK (STFC Daresbury Laboratory, Warrington, 2009). 